\title{
Dengue: Update on Epidemiology
}

\author{
Mary Elizabeth Wilson • Lin H. Chen
}

Published online: 5 December 2014

(C) Springer Science+Business Media New York 2014

\begin{abstract}
The epidemiology of dengue fever has undergone major shifts in recent decades. The global distribution has expanded to include more geographic areas. The intensity of transmission and the severity of infections have increased in areas where infection was already endemic. Multiple studies provide a clearer picture of the epidemiology and allow mapping of its distribution and change over time. Despite major efforts to control transmission, competent vectors now infest most tropical and subtropical regions; Aedes albopictus, also a competent vector, is able to survive in temperate areas, placing parts of Europe and North America at risk for local transmission. Many research teams in dengue-endemic areas are working to identify key local weather, vector, and other variables that would allow prediction of a likely epidemic early enough to permit interventions to avert it or blunt its impact.
\end{abstract}

Keywords Dengue $\cdot$ Epidemiology $\cdot$ Epidemic .

Hemorrhagic fever · Travel · Chikungunya $\cdot$ Mosquito

Vector - Emerging infection - Co-infection · Disease burden . Mapping $\cdot$ Climate change $\cdot$ Aedes aegypti $\cdot$ Aedes albopictus .

This article is part of the Topical Collection on Tropical, Travel and Emerging Infections

M. E. Wilson

University of California, San Francisco, CA, USA

M. E. Wilson

Harvard School of Public Health, Boston, MA, USA

M. E. Wilson $(\square)$

550 16th St, Global Health Sciences, Mission Hall, Box 1224,

San Francisco, CA 94158, USA

e-mail: mewilson@hsph.harvard.edu

L. H. Chen

Harvard Medical School, Travel Medicine Center, Mount Auburn

Hospital, Cambridge, MA, USA
Seroptyes $\cdot$ Zika virus $\cdot$ Surveillance $\cdot$ Transmission $\cdot$ Models $\cdot$ Forecasting outbreaks $\cdot$ Air travel

\section{Introduction}

The epidemiology of dengue is dynamic. Transmission is occurring in areas where infection had been absent or never previously documented, reappearing in areas without transmission for years to decades, and intensifying (more cases and more severe disease) in areas with endemic and epidemic transmission. This paper will describe the current epidemiology of dengue and some of the factors that influence it. It will also describe some of the new tools and approaches being used to try to anticipate and predict the epidemiology so that health officials can better prepare for future needs.

\section{Mapping and Estimating Disease Burden of Dengue}

Three papers provide analyses from a global perspective. Brady and colleagues bring together all available information about dengue occurrence to develop an evidence consensus map of disease that also identifies geographic areas with uncertain dengue status [1]. They drew upon materials from 1960 through 2012 including reports from health organizations (e.g., WHO, CDC, Gideon), peer-reviewed evidence, case data (including reports of infections in returned travelers), and supplementary evidence (e.g., Google news archives, HealthMap, others). They assigned a relative measure of certainty re presence or absence of dengue by country. Globally, they found evidence for dengue transmission in 128 countries [1]. At the same time, WHO maps showed dengue transmission in only 100 countries. Most of the newly defined transmission areas identified by Brady and colleagues 
were in Africa; these were also areas with the highest levels of uncertainty about the data.

In a series of maps, Messina and colleagues depict the global distribution of dengue from 1943 to 2013 and the global expansion by dengue type. By time period (usually decade), the maps show the distribution in sequence for each of the dengue serotypes. Maps also consolidate data by serotype to show the expansion of areas with circulation of multiple different serotypes [2]. These visually convey the impressive expansion of dengue over seven decades by showing the cumulative number of dengue types by geographic area by decade.

Bhatt and colleagues used maps of the global distribution of dengue and then paired risk maps with data from other studies to infer the burden of dengue in 2010 globally and regionally [3]. They estimated that there are 390 million dengue infections per year with 96 million having clinical manifestations, more than three times higher than WHO estimates. The authors estimate that Asia bears $70 \%$ of the burden (India alone is estimated to bear $34 \%$ of the global burden), Africa $16 \%$, and the Americas $14 \%$ (with more than half of that burden borne by Brazil and Mexico). They note the importance of mild and inapparent infections as a source of virus that can be transmitted; these undiagnosed infections also shape population patterns of immunity and dynamics of spread.

In recent years, advances in molecular methods have led to more reliable laboratory confirmation of dengue virus infections and also aided in the study of dengue epidemiology described in this review [4-6]. Molecular methods, specifically reverse-transcription polymerase chain reaction (RT-PCR) assays, have become widely applied and appear to have overtaken plaque reduction neutralization tests in confirming dengue infections. Moreover, amplification and sequencing of viral genes following extraction of dengue RNA has enabled comparison with available dengue virus genomes. These techniques have identified clades and genotypes within a serotype and determined genetic relatedness of the viruses [5, 7]. Based on these phylogenetic analyses, further understanding of dengue virus origin, movement, and distribution has become possible $[6,8,9]$.

\section{Americas}

Although massive epidemics occurred in the mainland USA in the past, only sporadic cases related to local transmission were documented in recent decades [10]. Since 2009, a focus of ongoing transmission has been established in Key West, Florida [11]. The number of cases has been small. Sequence data from DENV-1 viruses in Florida confirm endemic transmission of a distinct and predominant sublineage of the American-African genotpe for $>2$ years [5]. Viruses causing travel-related infections identified from other parts of Florida had Central American lineage, most closely related to DENV in Nicaragua and Mexico [12].

Dengue in the Americas is increasing. In the last 30 years in the Americas, the number of cases of dengue hemorrhagic fever has increased more than eightfold $[13,14]$. In Peru, a dengue outbreak during 2010 and 2011 was unprecedented in its magnitude and severity. Whole-genome sequencing documented that the responsible virus was a DENV-2 belonging to lineage II of Southeast Asian/American genotype that differed from the DENV-2 virus that had been circulating in the region since 1990 [9]. In this case, it appeared that the introduction of a new virus lineage contributed to the changed epidemiology.

Under some climate change scenarios for the northeastern USA, the distribution of Aedes albopictus, an invasive vector competent to transmit dengue, would expand [15]. Currently, the northernmost location of Ae. albopictus in the USA is New Jersey, southern NY, and Pennsylvania. Rochlin and colleagues have modeled potential range under different climate change scenarios [15]. They found that the land area environmentally suitable for Ae. albopictus could potentially increase from the current $5-16 \%$ up to $43-49 \%$ by the end of the century. Because large population centers are located in this region of the country, as many as 30 million residents could live in areas infested with Ae. albopictus. This mosquito is also competent to transmit chikungunya, a virus recently introduced into the Western Hemisphere [16], and other arboviruses, such as Zika virus.

Araujo and colleagues traced the origin and evolution of DENV-3 in Brazil. Introductions of DENV-3 from Caribbean to Brazil are relatively frequent. They note that DENV-3 genotype III probably emerged in mid 1970s in the Indian sub-continent and then spread. It was first detected in the Americas during outbreaks of severe and complicated dengue in Nicaragua and Panama in 1994 and has subsequently spread through the region [17].

DENV-4 reemerged in Brazil in 2010 in the state of Roraima in the Northwest. It subsequently reached Rio de Janeiro and has spread widely $[6,18]$. An analysis of the spatial dynamics of DENV-1, DENV-2, and DENV-3 in Brazil found that the introduction of new lineages occurred at about 7-10-year intervals. The origin was most often the Caribbean region. New lineages were introduced into the North and Northeast and then dispersed. The authors concluded that air transport of humans and/or vector mosquitoes determined the spread of dengue virus in Brazil [19]. In general, in Brazil, between 2000 and 2010, the epidemiological pattern was complex but with an overall increase in the number of cases and a rise in the proportion of severe cases [20, 21].

Much attention was focused on Brazil during the FIFA World Cup events of 2014. Although an analysis of GeoSentinel data found that dengue accounted for $31 \%$ of 
all acute systemic febrile illnesses with a specific cause identified in travelers who returned from Brazil, the majority of the dengue cases occurred in the months of January through May, before the cooler months when the World Cup events were held [22]. Other investigators used models to anticipate the size of the dengue burden in travelers to Brazil [23, 24]. Fortunately, no major outbreaks were reported among visitors to Brazil during and immediately after the World Cup events.

\section{Middle East}

Several papers describe the emergence of dengue in the Middle East $[25,26]$, especially in the Arabian peninsula. Large epidemics have been reported from Yeman and Saudi Arabia since the mid 1990s. Deaths from dengue hemorrhagic fever and dengue shock syndrome have been reported as well. Although rainfall is low and climatic conditions in the region are unfavorable for Aedes aegypti, the vector is present in some of the countries. Construction sites and temporary settlements may provide conditions suitable for breeding. Other risk factors include increased travel and trade (large immigrant work force from dengue-endemic countries; travel associated with pilgrims to the Hajj) and increasing urbanization. In Saudi Arabia, 4411 dengue cases were reported in 2013, fourfold higher than in 2012, with peak transmission in May [27]. Dengue viruses 1-3 have been reported in the region. As has been reported in other regions (including Africa, Asia, Pacific Islands), co-circulation of dengue and chikungunya viruses has been documented in Yemen [28].

\section{Africa}

Many recent reports document presence of dengue in Africa $[1,4,29]$. Since 1960, dengue has been reported in 32 African countries, though less certainty exists around the burden and distribution in Africa than in other areas. All four serotypes have been found in Africa although infections with DENV-2 have been most common. In Gabon, in recent years, three serotypes circulated simultaneously [30]. Co-circulation of dengue and chikungunya viruses has occurred and coinfection in a traveler documented [31]. Available data come from local studies, including serosurveys, reports of outbreaks, and entomological studies [30, 32]. Reports of dengue infections in travelers returning to dengue-non-endemic areas have also confirmed outbreaks in some areas and provided early alerts in some instances $[4,31,33]$.

In 2013, an expert panel was convened to review dengue situation in Africa; their recommendations were recently published [34]. To better assess the burden of dengue in Africa, they recommend that diagnostics be made more widely available throughout Africa and that networks collaborate to produce and collect this type of data. Better assessment of the burden is needed to inform policy decisions around vector control and health services. At least two competent dengue vectors, Ae. aegypti and Ae. albopictus, are present in Africa.

Some investigators have proposed that protective genetic variants could partially explain a lower burden of dengue in Africa (see below); however, severe and complicated infections have been documented in Africa. The common occurrence of malaria and other febrile illnesses and lack of good diagnostics in many areas probably contribute to infrequent diagnosis of dengue fever.

\section{Asia}

Transmission of dengue remains widespread in Asia with frequent, locally severe outbreaks. Notably, local transmission of dengue has been reported recently in Japan [35]. This included a cluster of infections in Tokyo [36].

The first suspected outbreak in Shenzhen, China, in 2010, was confirmed through RT-PCR, virus isolation and culture, and nucleic acid sequencing as well as serology [37]. Investigators found the causative DENV-1 to resemble those recently isolated from Malaysia and Singapore. Since no imported cases were reported during this outbreak, they postulated that mosquito eggs from Southeast Asia may have been imported and led to vertical transmission of the virus [37]. Elsewhere, in Guangdong Province, China, which is known to have dengue virus transmission, high-epidemic areas appeared to be in the Pearl River Delta and the Han River Delta regions [38].

A cross-sectional serosurvey involving 1000 subjects (3574 years old) in Malaysia found high rates of IgG seropositivity (>90\%). Seroprevalence was similar among all ethnic groups and both genders. It was also similar among rural and urban residents suggesting that in Malaysia, dengue is widespread and not predominantly an urban infection [39].

A phylogeographic analysis of 751 complete genome sequences of DENV-1 from urban and rural Vietnam found that various lineages of DENV-1 show spatial clustering in Vietnam [40]. This study found that DENV-1 tends to move from urban to rural populations; densely populated regions within Ho Chi Minh City were major transmission foci-a finding with implications for control measures. In some areas, a lineage moved less than $20 \mathrm{~km} /$ year, suggesting mosquitomediated dispersal. Interestingly, a multi-center study in Asia found chikungunya and Salmonella typhi infections to be even more common than dengue [41].

\section{Pacific Islands}

A dengue outbreak on Kosrae in the Federated States of Micronesia occurred in 2012-2013 [42]. The only previously 
documented outbreak was in 1998. The recent outbreak was intense; almost $4 \%$ of the residents of the island were hospitalized with suspected dengue infection. The cumulative incidence of infection during the 5.5-month outbreak period was 31 laboratory-confirmed and 110 suspected infections per 100,000 population. Although both Ae aegypti and Ae. albopictus infested the island, Ae. albopictus predominated in a vector survey making up $>90 \%$ of the larval mosquito samples. In one municipality, one third of all used tires were found to have vector mosquito larvae.

Dengue viruses 1 and 3 circulated in the South Pacific island of French Polynesia in 2013-2014 at the same time that Zika virus was implicated in a massive outbreak, causing an estimated 28,000 cases ( $11 \%$ of the population) [43, 44]. Zika virus is also transmitted by Ae. aegypti and Ae. albopictus.

\section{Europe}

Occasional sporadic cases of local dengue transmission have occurred in mainland Europe in France and Croatia [45, 46]. Ae. albopictus has been established in southern France since 2007 and is found in large parts of southern Europe. Ae. aegypti is also present in parts of Europe [47]. In a period of less than 4 months during the summer of 2014, 370 confirmed cases of dengue and chikungunya infection were reported in mainland France in areas colonized by Ae. albopictus [48]. Schaffner and Mathis review the current distribution of dengue vectors in Europe and their potential future distributions [49], which could expand with changes in temperature and rainfall. Several areas are at risk for the potential introduction of chikungunya and Zika viruses as well as dengue transmission.

In 2012, a major outbreak of dengue occurred on the island of Madeira [50]. The DENV-1 outbreak involving $>2100$ cases began in October 2012 and continued through March 2013. Cases were introduced into 13 European countries by travelers who had become infected in Madeira. An analysis of travelers to Madeira in 2012 found that they originated from 29 dengue-endemic countries; overall, almost $90 \%$ originated in Venezuela or Brazil. Genetic-sequencing studies showed the virus identified in Madeira outbreak was most closely related to viruses circulating in Venezuela, Brazil, and Colombia. Based on travel volume and dengue incidence in countries of traveler origin, Wilder-Smith and colleagues concluded that Venezuela was most likely the country of origin of the virus causing the outbreak [51].

\section{Surveillance and Warning Systems}

Interest is strong in developing effective low-cost early warning systems that can predict dengue epidemics and allow interventions that can blunt the size of epidemics [52]. In Peru, investigators used a novel prediction method utilizing fuzzy association rule mining. Inputs included clinical, meteorological, climatic, and socio-political data. They used these data to predict future dengue incidence as either high or low. They note that the same method could be used in other geographical regions [53]. In Colombia, investigators developed models-environment-based, multivariate, and autoregressive forecast models - that allow dengue outbreaks to be anticipated from 2 weeks to 6 months before they would occur. They used epidemiological and meteorological data for urban Cali, Colombia, from Jan 2000 to Dec 2011. They found outbreaks occurred during warm-dry periods with daily temperatures between 18 and $32{ }^{\circ} \mathrm{C}$ [54].

In Thailand, Campbell and colleagues assessed relationship between weather and dengue transmission [55]. They found that the temperature defined the range within which transmission was viable. Within a permissive temperature range, humidity amplified potential transmission. They found that in Thailand, $80 \%$ of the severe dengue cases occurred when mean humidity was $>75 \%$ and mean temperature was 27 $29.5^{\circ} \mathrm{C}[55]$.

In areas with intense ongoing transmission, studies have identified humidity as a key feature among climatic variables in explaining the seasonal patterns of infection. In Singapore, $\mathrm{Xu}$ and colleagues found that absolute humidity was a better predictor for modeling dengue incidence than other weather variables [56]. Mean temperature also correlated with incidence but absolute humidity had a more stable impact on dengue incidence [56]. Many recently published studies have focused on finding locally relevant data to provide early warnings [52, 57-59]. Daily temperature fluctuations are also relevant. Mosquitoes live longer and are more likely to become infected when temperature fluctuations are not extreme [60].

Liu-Helmersson and colleagues have pointed to the importance of diurnal temperature range in the dengue epidemic potential [61]. Many previous studies have focused only on the mean temperature values.

In coastal Ecuador, researchers identified non-climate as well as climate drivers that could be monitored to provide a predictive lead for forecasting dengue epidemics [62]. In their model, the percent of households with immature Ae. aegypti was also significant in predicting outbreaks. Other investigators have looked to web searches and social media to monitor dengue epidemiology [63-65].

A study in Vietnam assessed host and viral parameters that influence DENV transmission from infected humans to $A e$. aegypti mosquitoes [66]. They found that high early plasma virus levels were associated with longer duration infectiousness of the mosquitoes. Hospitalized patients had higher viremia levels than ambulatory dengue cases but virus levels in ambulatory cases were still sufficient to infect mosquitoes. 
A detailed longitudinal study in Peru was able to provide serotype-specific estimates of the dengue virus force of infection (the per capita risk of a susceptible person being infected) [67]. The study showed that the force of infection varied markedly by time and by serotype. Transmissibility varied significantly by serotype as did the capacity (by serotype) to survive over the winter. The study is important in highlighting how cross-immunity shapes incidence patterns [68]. Presence of asymptomatic infections makes it difficult to track the prevalence of active infection (and presence of virus).

Many dengue investigators have speculated that host genetics might be one factor that influences severity of dengue infections. A recent study supports earlier findings suggesting that African ancestry protects against severe outcomes in dengue [69]. The most recent study was carried out in Colombia and looked at a population with European, Native American, or African ancestors. Based on extracted DNA, they looked at 30 Ancestry Informative Markers (AIM) to assess the mix of genetic ancestry for each of the patients with documented dengue infection. Having fewer African ancestry markers was significantly associated with more severe clinical dengue.

The WHO issued new guidelines for dengue classification in 2009. An analysis of their use based on a systematic literature review concluded that the new classification has clear advantages for clinical use. For those doing epidemiological and pathogenesis research, the new guidelines offer the advantages of international comparability, ease of application, and increased sensitivity for identifying severe dengue [70].

\section{Novel Means of Transmission}

Transmission of dengue virus by routes other than the mosquito vector are well established, including blood transfusion and organ transplantation and needlestick. A case report describes transmission of dengue virus to a peripheral blood stem cell recipient by a donor who had recently traveled to Sri Lanka, a dengue-endemic area. On the day of apheresis, the donor had signs of a respiratory infection; dengue was not initially suspected [71].

\section{Travelers}

Dengue is an important cause of febrile illness in travelers, and identification of infections in travelers helps to define the global epidemiology of dengue [72]. In the USA, an upward trend in hospitalizations for dengue has been observed [73]. Ratnam and colleagues have recently reviewed dengue and international travel [74]. In a study on Australian travelers, seroconversion to dengue occurred in $1.0 \%$, an estimated incidence of 3.4 infections per 10,000 days of travel in this cohort; travel to India and China were implicated and all cases were subclinical [75]. Among travelers to dengue-endemic countries who had a pre-travel evaluation in the Boston area, $19 \%$ of those who were born or had lived in dengue-endemic countries had anti-dengue IgG antibody by ELISA and $12 \%$ had antibodies by PRNT [76]. Antibody positivity was associated with duration of living in dengue-endemic countries. Among the antibody-positive individuals, $85 \%$ had no history of clinical dengue.

To assess which countries pose the highest dengue risk to travelers, researchers analyzed data on confirmed dengue cases reported to the Swedish Institute for Communicable Disease Control [77]. They also had numbers of Swedish visitors to each country for each year during the study period. Most of the 864 infections were acquired in one of six Southeast Asian countries. They noted that attack rates appeared to be increasing over the period from 1995 to 2010 . Attack rates per 100,000 Swedish travelers per year were highest for Sri Lanka (45.3), Bangladesh (42.6), and El Salvador (33.4). The attack rate for Thailand, a common tourist destination, was 13.6/100,000.

Massad and colleagues developed a mathematical model to quantify the risk of acquiring dengue by a non-immune traveler to Thailand [78]. They calculated that a traveler arriving during high-transmission season and staying for 7 days had $0.2 \%$ risk of acquiring dengue. This increased to $0.81 \%$ for someone staying 30 days. The results of the model are similar to incidence rates reported in previously published prospective seroconversion studies on travelers. The authors note that models may help travel medicine providers provide estimates of risk for various travel scenarios [78].

Dengue infections imported by travelers can initiate local epidemics, but only when meteorological conditions are favorable [Shang]. Shang and colleagues in Taiwan note the importance of early case detection of imported cases and the need to integrate meteorological data with surveillance data to allow interventions to avert outbreaks [79].

Stoddard and colleagues conducted a large longitudinal cohort study in Iquitos, Peru, to assess the role of human movement in the epidemiology of dengue in a community [80]. They found that houses are the likely place of exposure in this community. Houses visited by infected individuals were at risk for infection. Movement of infected and susceptible individuals shaped the epidemiology. They concluded that transmission is shaped by social connections and that this knowledge should be considered in vector control strategies.

\section{Conclusion}

In summary, an abundance of new studies provide new insights into the epidemiology and transmission of dengue infections globally [81]. Despite new information and better 
understanding of transmission dynamics, the global situation with dengue continues to worsen with expansion of affected areas with circulation of one or more dengue serotypes.

\section{Compliance with Ethics Guidelines}

Conflict of Interest Mary Wilson has no conflicts of interest. Lin Chen reports personal fees from Shoreland Inc, another from GSK, and personal fees from Elsevier Publishing, another from Wiley Publishing, outside the submitted work.

Human and Animal Rights and Informed Consent This article does not contain any studies with human or animal subjects performed by the author.

\section{References}

Papers of particular interest, published recently, have been highlighted as:

- Of importance

•. Of major importance

1.• Brady OJ, Gething PW, Bhatt S, et al. Refining the global spatial limits of dengue virus transmission by evidence-based consensus. PLoS Negl Trop Dis. 2012;6(8):e1760. doi:10.1371/journal.pntd. 0001760. Authors created maps that indicate level of uncertainty about transmission. Paper includes map of 128 countries with good evidence of dengue occurrence.

2.• Messina JP, Brady OJ, Scott TW, et al. Global spread of dengue virus types: mapping the 70 year history. Trends Microbiol. 2014;22:138-46. doi:10.1016/j.tim.2013.12.011. PubMed Series of maps depict the global expansion of dengue viruses by serotype.

3.• Bhatt S, Gething PW, Brady OJ, et al. The global distribution and burden of dengue. Nature. 2013;496:504-7. Excellent overview of global distribution and burden of dengue.

4. CDC. Ongoing dengue epidemic- Angola, June 2013. MMWR 2013;62:early release. June 17, 2103.

5. Munoz-Jordal J, Santiago GA, Margolis H, Stark L. Genetic relatedness of dengue viruses in Key West, Florida, USA, 2009-2010. Emerg Infect Dis. 2013;19(4):652-4.

6. Villabona-Arenas CJ, de Oliveira JL, Capra CS, et al. Detection of four dengue serotypes suggests rise in hyperendemicity in urban centers of Brazil. PLoS Negl Trop Dis. 2014;8(2):e2620. doi:10. 1371/journal.pntd.0002620.

7. Teoh BT, Sam SS, Tan KK, et al. Dengue virus type 1 clade replacement in recurring homotypic outbreaks. BMC Evol Biol. 2013;13:213.

8. Koo C, Nasir A, Hapuarachchi HC, et al. Evolution and heterogeneity of multiple serotypes of dengue virus in Pakistan, 2006-2011. Virol J. 2013;10:275.

9. Williams M, Mayer SV, Johnson WL, et al. Lineage II of Southeast Asian/American DENV-2 is associated with a severe dengue outbreak in the Peruvian Amazon. Am J Trop Med Hyg. 2014;91(3): 611-20.

10. Centers for Disease Control and Prevention. Locally acquired dengue-Key West, Florida, 2009-2010. MMWR Morb Mortal Wkly Rep 2010. 2010;59:577-81.

11. Radke EG, Gregory CJ, Kintziger KW, et al. Dengue outbreak in Key West, Florida, USA, 2009. Emerg Infect Dis. 2012;18(1):135-7.
12. Shin D, Richards SL, Alto BW, et al. Genome sequence analysis of dengue virus 1 isolated in Key West, Florida. PLoS One. 2013;8(9): e74582.

13. San Martin JL, Braithwaite O, Zambrano B, et al. The epidemiology of dengue in the Americas over the last three decades: a worrisome reality. Am J Trop Med Hyg. 2010;82:128-35.

14. Dick OB, San Martin JL, Montoya RH, et al. The history of dengue outbreaks in the Americas. Am J Trop Med Hyg. 2012;87(4):584-93.

15. Rochlin I, Ninivaggi DV, Hutchinson ML, Farajollahi A. Climate change and range expansion of the Asian tiger mosquito (Aedes albopictus) in Northeastern USA: implications for public health practitioners. PLoS One. 2013;8(4):e60874. doi:10.1371/journal. pone. 0060874 .

16. Nasci RS. Movement of chikungunya virus into the Western Hemisphere. Emerg Infect Dis. 2014;20(8):1394-95.

17. JMGd A' j, Bello G, Romero H, Nogueira RMR. Origin and evolution of dengue virus type 3 in Brazil. PLoS Negl Trop Dis. 2012;6(9):e1784. doi:10.1371/journal.pntd.0001784.

18. Nogueira RM, Eppinghaus AL. Dengue virus type 4 arrives in the state of Rio de Janeiro: a challenge for epidemiological surveillance and control. Mem Inst Oswaldo Cruz. 2011;106:255-6.

19. Nunes MRT, Palacios G, Faria NR, et al. Air travel is associated with intracontinental spread of dengue virus serotypes 1-3 in Brazil. PLoS Negl Trop Dis. 2014;8(4):e2769. doi:10.1371/ journal.pntd.0002769.

20. Teixeira MG, Siqueira Jr JB, Ferreira GLC, et al. Epidemiological trends of dengue disease in Brazil (2000-2010): a systematic literature search and analysis. PLoS Negl Trop Dis. 2013;7(12):e2520. doi:10.1371/journal.pntd.0002520.

21. Rodriguez-Barraquer I, Cordeiro MT, Braga C, et al. From reemergence to hyperendemicity: the natural history of the dengue epidemic in Brazil. PLoS NTD. 2011;5(1):e935.

22. Wilson ME, Chen LH, Han PV, GeoSentinel Surveillance Network, et al. Illness in travelers returned from Brazil: the GeoSentinel experience and implications for the 2014 World Cup and the 2016 Summer Olympic Games. Clin Infect Dis. 2014;58(15 May):134756. doi:10.1093/cid/ciu122.

23. Massad E, Nurattini MN, Ximenes R, et al. Dengue outlook for the World Cup in Brazil. Lancet Infect Dis 2014; published online 12 June 2014. Letter

24. Van Panhuis WG, Hyun S, Blaney K, et al. Risk of dengue for tourists and teams during the World Cup 2014 in Brazil. PLoS NTD. 2014;8(7):e3063.

25.• Amarasinghe A, Letson GW. Dengue in the Middle East: a neglected and emerging disease of importance. Commentary. Trans R Soc Trop Med Hyg. 2012;106:1-2. Highlights some of factors associated with transmission in an area previously considered low risk.

26. Bin G, Amarasingh A, Letson W. Dengue outbreak in Hadramont, Yemen, 2010: an epidemiological perspective. Am J Trop Med Hyg. 2012;86:1072-6. doi:10.4269/ajtmh.2012.11-0723.

27. Aziz AT, Al-Shami SA, Mahyoub JA, et al. An update on the incidence of dengue gaining strength in Saudi Arabia and current control approaches for its vector mosquito. Parasite Vectors. 2014;7(1):258.

28. Rezza G, El-Sarif G, Faggioni G, et al. Co-circulation of dengue and chikungunya viruses, Al Hudaydah, Yemen, 2012. Emerg Infect Dis. 2014;20(8):1351-54.

29. Faye O, Ba Y, Talla C, Diallo D, Chen R. Urban epidemic of dengue virus serotype 3 infection, Senegal, 2009. Emerg Infect Dis. 2014;20:456-9. doi:10.3201/eid2003.121885 PubMed.

30. Caron M, Grard G, Paupy C, et al. First evidence of simultaneous circulation of three different dengue virus serotypes in Africa. PLoS One. 2013;8(10):e78030. doi:10.1371/journal.pone.0078030. 
31. Parreira R, Centeno-Lima S, Lopes A, et al. Dengue virus serotype 4 and chikungunya virus coinfection in a traveller returning from Luanda, Angola, January 2014. Euro Surveill. 2014;19(10):pii= 20730. Available online: http://www.eurosurveillance.org/ ViewArticle.aspx?ArticleId $=20730$

32. Sutherland LJ, Cash AA, Huang Y-JS, Sang RC, Malhotra I, Moormann AM, et al. Serologic evidence of arboviral infections among humans in Kenya. Am J Trop Med Hyg. 2011;85(1): 158-61.

33. Schwartz E, Meltzer E, Mendelson M, et al. Detection on four continents of dengue fever cases related to an ongoing outbreak in Luanda, Angola, March to May 2013 . Euro Surveill. 2013;18(21): pii=20488. Available online: http://www.eurosurveillance.org/ ViewArticle.aspx?ArticleId=20488

34. Jaenisch T, Junghanss T, Wills B, et al. Dengue expansion in Africa - not recognized or not happening? Emerg Infect Dis [Internet]. 2014 Oct [date cited].

35. Schmidt-Chanasit J, Emmerich P, Tappe D, et al. Autochthonous dengue virus infection in Japan imported into Germany, September 2013. Euro Surveill. 2014;19(3):pii=20681. Available online: http://www.eurosurveillance.org/ViewArticle.

36. ProMED post. Dengue_Japan: (ST) 20140828.2731079. 2014.

37. Yang F, Guo GZ, Chen JQ, et al. Molecular identification of the first local dengue fever outbreak in Shenzhen city, China: a potential imported vertical transmission from Southeast Asia? Epidemiol Infect. 2014;142(2):225-33

38. Fan $\mathrm{J}$, Lin $\mathrm{H}$, Wang $\mathrm{C}$, et al. Identifying the high-risk areas and associated meteorological factors of dengue transmission in Guangdong Province, China from 2005 to 2011. Epidemiol Infect. 2014;142(3):634-43.

39. Mohammad Azami NA, Salleh SA, Neoh HM, et al. Dengue epidemic in Malaysia: not a predominately urban disease any more. BMC Res Notes. 2011;4:216.

40. Raghwani J, Rambaut A, Holmes EC, et al. Endemic dengue associated with the co-circulation of multiple viral lineages and localized density-dependent transmission. PLoS Pathog. 2011;7: e1002064

41. Capeding MR, Chua MN, Hadinegoro SR, et al. Dengue and other common causes of acute febrile illness in Asia: an active surveillance study in children. PLoS Negl Trop Dis. 2013;7(7):e2331.

42. Centers for Disease Control and Prevention (CDC). Dengue outbreak-Federated States of Micronesia, 2012-13. MMWR Morb Mortal Wkly Rep. 2013;62:570-3.

43. Cao-Lormeau VM, Roche C, Musso D, et al. Dengue virus type 3, South Pacific Islands, 2013. Emerg Infect Dis. 2014;20(6):1034-6.

44. Musso D, Nhan T, Robin E, et al. Potential for Zika virus transmission through blood transfusion demonstrated during an outbreak in French Polynesia, November 2013 to February 2014. Eurosurveillance 2014;19(14). 10 April.

45. Marchand E, Prat C, Jeannin C, et al. Autochthonous case of dengue in France, October 2013. Euro Surveill. 2013;18(50):pii= 20661. Available online: http://www.eurosurveillance.org/ ViewArticle.aspx?ArticleId=20661

46. Gjenero-Margan I, Aleraj B, Krajcar D, et al. Autochthonous dengue fever in Croatia, August-September 2010. Euro Surveill. 2011;16(9):pii=19805. Available from: http://www. eurosurveillance.org/ViewArticle.aspx?ArticleId=19805

47. European Centre for Disease Prevention and Control [ECDC]. VBORNET Maps - Mosquitoes. Stockholm: ECDC. Available from: http://ecdc.europa.eu/en/activities/diseaseprogrammes/ emerging and_vector_borne_diseases/Pages/VBORNET_maps. aspx

48. Schaffner F, Fontenille D, Mathis A. Autochthonous dengue emphasizes the threat of arbovirosis in Europe. Lancet infect Dis 2014. Published online 11 Sept 2014.
49. Schaffner F, Mathis A. Dengue and dengue vectors in the WHO European Region: past, present, and scenarios for the future. Lancet Infect Dis 2014; published online 27 August.

50. Sousa CA, Clairouin M, Seixas G, et al. Ongoing outbreak of dengue type 1 in the Autonomous Region of Madeira, Portugal: preliminary report. Euro Surveill. 2012;17(49):pii=20333. Available online: http://www.eurosurveillance.org/ViewArticle. aspx?ArticleId $=20333$

51. Wilder-Smith A, Quam M, Sessions O, et al. The 2012 dengue outbreak in Madeira: exploring the origins. Euro Surveill. 2014;19(8):pii=20718. Available online: http://www. eurosurveillance.org/ViewArticle.aspx?ArticleId=20718

52. Descloux E, Mangeas M, Menkes CE, et al. Climate-based models for understanding and forecasting dengue epidemics. PLoS Negl Trop Dis. 2012;6(2):e1470. doi:10.1371/journal.pntd.0001470.

53. Buczak AL, Koshute PT, Babin SM, et al. A data-driven epidemiological prediction method for dengue outbreaks using local and remote sensing data. BMC Med Inf Decis Making. 2012;12:124.

54. Eastin MD, Delmelle E, Casas I, et al. Intra- and interseasonal autoregressive prediction of dengue outbreaks using local weather and regional climate for a tropical environment in Colombia. Am J Trop Med Hyg. 2014;91(3):598-610.

55. Campbell KM, Lin DC, Iamsirithaworn S, Scott TW. The complex relationship between weather and dengue virus transmission in Thailand. Am J Trop Med Hyg. 2013;89(6):1066-80. Good discussion of the interaction of temperature and humidity in dengue transmission.

56. $\mathrm{Xu} \mathrm{H}-\mathrm{Y}, \mathrm{Fu} \mathrm{X}$, Lee $\mathrm{LKH}$, et al. Statistical modeling reveals the effect of absolute humidity on dengue in Singapore. PLoS Negl Trop Dis. 2014;8(5):e2805. doi:10.1371/journal.pntd.0002805.

57. Earnest A, Tan SB, Wilder-Smith A. Meteorological factors and El Niño Southern Oscillation are independently associated with dengue infections. Epidemiol Infect. 2012;140:1244-51.

58. Gomes AF, Nobre AA, Cruz OG. Temporal analysis of the relationship between dengue and meteorological variables in the city of Rio de Janeiro, Brazil, 2001-2009. Cad Saude Publica. 2012;28: 2189-97.

59. Massad E, Pinto E, Coelho M, Oliver L. The influence of climate variables on dengue in Singapore. International Journal of Environmental Health Research, First published on: 09 May 2011 (iFirst)

60. Lambrechts L, Paaijmans KP, Fansiria T, et al. Impact of daily temperature fluctuations on dengue virus transmission by Aedes aegypti. Proc Natl Acad Sci U S A. 2011;108:7460-5.

61. Liu-Helmersson J, Stenlund H, Wilder-Smith A, Rocklov J. Vectorial capacity of Aedes aegypti: effects of temperature and implications for global dengue epidemic potential. PLoS One. 2014;9(3):e89783. doi:10.1371/journal.pone.0089783.

62. Stewart-Ibarra AM, Lowe R. Climate and non-climate drivers of dengue epidemics in southern coastal Ecuador. Am J Trop Med Hyg. 2013;88(5):971-81.

63. Chan EH, Sahai V, Conrad C, Brownstein JS. Using web search query data to monitor dengue epidemics: a new model for neglected tropical disease surveillance. PLoS Negl Trop Dis. 2011;5:e1206.

64. Gluskin RT, Johansson MA, Santillana M, Brownstein JS. Evaluation of internet-based dengue query data: Google dengue trends. PLoS Negl Trop Dis. 2014;8(2):e2713. doi:10.1371/ journal.pntd.0002713.

65. Hoen AG, Keller M, Verma AD, et al. Electronic event-based surveillance for monitoring dengue. Latin America Emerg Infect Dis. 2012;18(7):1147-50.

66. Nguyen MN, Kien DTH, Tuan V, et al. Host and viral features of human dengue cases shape the population of infected and infectious Aedes aegypti mosquitoes. PNAS. 2013;110(22):9072-7. Important study assessing relationship between viremia and infected mosquitoes. 
67.• Reiner RC, Stoddard ST, Forshey BM, et al. Time-varying, serotype-specific force of infection of dengue virus. PNAS. 2014;111:E2694-702. Longitudinal study that assesses force of infection for various serotypes.

68. Ferguson NM, Cummings DAT. How season and serotype determine dengue transmissibility. PNAS. 2014;111:9370-71.

69. Chacon-Duque JC, Adhikari K, Avendano E, et al. African genetic ancestry is associated with a protective effect on dengue severity in Colombian populations. Infect Genet Evol. 2014;27:89-95.

70. Horstick O, Jaenisch T, Martinez E, et al. Comparing the usefulness of the 1997 and 2009 WHO dengue case classification: a systematic literature review. Am J Trop Med Hyg. 2014;91(3):621-34.

71. Punzel M, Korukluoglu G, Caglayik EY, et al. Dengue virus transmission by blood stem cell donor after travel to Sri Lanka; Germany, 2013. Emerg Infect Dis 2014;20(8):

72. Domingo C, Niedrig M, Gasco'n J, et al. Molecular surveillance of circulating dengue genotypes through European travelers. J Travel Med. 2011;18:183-90.

73. Streit JA, Yang M, Cavanaugh JE, Polgreen PM. Upward trend in dengue incidence among hospitalized patients, United States. Emerg Infect Dis. 2011 May; [Epub ahead of print]
74. Ratnam I, Leder K, Black J, Torresi J. Dengue fever and international travel. J Travel Med. 2013;20:384-93. Nice review of dengue in travelers.

75. Ratnam I, Black J, Leder K, et al. Incidence and seroprevalence of dengue virus infections in Australian travellers to Asia. Eur J Clin Microbiol Infect Dis. 2012;31(6):1203-10.

76. Sanchez-Vegas C, Hamer DH, Chen LH, et al. Prevalence of dengue virus infection in US travelers who have lived in or traveled to dengue-endemic countries. J Travel Med. 2013;20(6):352-60. doi:10.1111/jtm.12057.

77. Rocklov J, et al. Attack rates of dengue fever in Swedish travellers. Scand J infect Dis 2014;

78. Massad E, Rocklov J, Wilder-Smith A. Dengue infections in nonimmune travellers to Thailand. Epidemiol Infect. 2013;141:412-7.

79. Shang C-S, Fang C-T, Liu C-M, et al. The role of imported cases and favorable meteorological conditions in the onset of dengue epidemics. PLoS Negl Trop Dis. 2010;4(8):e775. doi:10.1371/ journal.pntd.0000775.

80. Stoddard ST, Forshey BM, Morrison AC, et al. House-to-house human movement drives dengue virus transmission. PNAS. 2013;110(3):994-9. doi:10.1073/pnas.1213349110.

81.• Simmons CP, Farrar JJ, Chau NV, Wills B. Dengue. N Engl J Med. 2012;366(15):1423-32. Excellent overview of current state of knowledge of all aspects of dengue. 